\title{
X-Ray Study of Interfacial Interactions in Highly Milled
}

\author{
Sn-Ge Powders
}

BNL- -47382

\author{
J.K.D.S. Jayanetti, S.M. Heald and Z. Tan \\ DE92 012518 \\ Brookhaven National Laboratory, Upton NY 11973
}

\begin{abstract}
We have studied possible structural changes occuring at the $\mathrm{Sn} / \mathrm{Ge}$ interface of highly milled $\mathrm{Sn} / \mathrm{Ge}$ composites. EXAFS and X-ray Diffraction measurements were made on mechanically milled powders having compositions ranging from 20 to 50 vol.\% Sn. X-ray diffraction measurements indicate the increasing amorphization of $\mathrm{Sn}$ as the $\mathrm{Sn}$ content is decreased. EXAFS results indicate that this amorphous phase is due to the formation of an $\alpha-S n / G e$ alloy. The EXAFS from this alloy did not change significantly at the $\mathrm{Sn}$ melting point. X-ray diffraction measurements made at room temperature show a systematic decrease in the intensity of $\mathrm{Sn}$ peaks and broadening of Ge peaks with the decreasing Sn content.
\end{abstract}

\section{INTRODUCTION}

Recently Jang and Koch $[1,2]$ by their $\mathbf{x}$-ray diffraction and DSC studies on mechanically milled $\mathrm{Sn} / \mathrm{Ge}$ powders have made some interesting observations. They have seen the possible depression of the melting point and broadening of the melting transition region of $\mathrm{Sn}$ with decreasing $\mathrm{Sn}$ content. Also when the Ge volume reaches a value where essentially all the $\mathrm{Sn}$ atoms are adjacent to the Ge particle surfaces, the $\mathrm{Sn}$ is said to be in a disordered, perhaps in an amorphous, state such that no melting transition is observed. According to the simple eutectic Ge-Sn equilibrium diagram there is essentially no solid solubility of $\mathrm{Ge}$ in $\mathrm{Sn}$ and very small $(<1$ at. \%) solubility of $\mathrm{Sn}$ in Ge. It is interesting to see whether there is another phase of $\mathrm{Sn}$, namely the cubic form $\alpha-\mathrm{Sn}$, as it reaches this disorderd state. It is known that Ge can stabilize the $\alpha$-Sn phase. [3] The motive behind our work is to identify this so called disordered phase or form structurally, using Extended $x$-ray absorption fine structure (EXAFS) which is a proven tool to study disordered materials because of its ability to investigate the local atomic structure of each of the components in a material. X-ray diffraction and Differential Scanning Calorimetry have also been used to support our results. In this paper we discuss some of the results we have obtained recently.

\section{EXPERIMENTAL}

Sn/Ge powder samples were made using a Brinkmann, Model MM2, Standard M:xer Mill operated in an Ar ambient. Powders (-100 mesh,99.999\% pure) were milled in a sealed hardened tool steel vial, using a steel ball. Loading and unloading of powders to the vial were done inside a dry box with moisture and oxygen content of $<5 \mathrm{ppm}$. Powders with varying $\mathrm{Sn} / \mathrm{Ge}$ volume ratios $(20,30$, 40 and 50 vol.\% Sn) were milled for nearly a constant time of about 32 hours. Differential Scanning Calorimetric analysis done on these samples produced results similar to those of Jang and Koch.

In order to prepare $x$-ray samples of proper absorption, the powders were diluted to proper amounts with carbon and packed in an array of slots made in a rectangular $\mathrm{Cu}$ plate and sealed in a container which has windows (Kapton) transparent to $x$-rays. Another sample was packed in a slotted Al plate using thin $\mathrm{Al}$ windows to perform the heating experiments. An attempt was made to make an $\alpha$-Sn standard by melting a piece of regular $\mathrm{Sn}(\beta-S n)$ with a very small amount of $\mathrm{Ge}(<0.5$ at. $\%)$ in a UHV chamber. In order to initiate the transformation the specimen was kept in a freezer at $30^{\circ} \mathrm{C}$. After two days the sample had transformed. It was ground to a fine powder and rubbed onto 
Source. Measurements were made in the transmission mode using a Si(311) double crystal monochromator. Sn K-edge (29200 eV) EXAFS measurements were made at temperatures of $10^{\circ} \mathrm{K}$, $100^{\circ} \mathrm{K}$ and $200^{\circ} \mathrm{K}$, in a rough vacuum. One sample $(20 \mathrm{vol} . \% \mathrm{Sn})$ was heated in a vacuum heating stage above the $\mathrm{Sn}$ melting point while measuring EXAFS at several temperatures.

The diffraction measurements were made at room temperature at beamline $\mathrm{X}-11 \mathrm{~B}$ using a modified Phillips goniometer with an INEL $120^{\circ}$ curved position sensitive detector. This configuration allowed us to acquire a complete diffraction pattern in about 3 minutes.

\section{RESULTS AND DISCUSSION}

Figure 1 shows the EXAFS data obtained at $10^{\circ} \mathrm{K}$ for powder samples of two differcnt compositions namely $50 \mathrm{vol} . \% \mathrm{Sn}$ and $20 \mathrm{vol} . \% \mathrm{Sn}$. We have compared them to the EXAFS of $\alpha$-Sn (our standard which has some $\beta$-Sn contribution) and $\beta$-Sn obtained at the same temperature. X-ray diffraction data shows (Figure 2 ) a decreasing relative intensity of the $\beta$-Sn peaks. No new diffraction peaks seem to evolve. This indicates that $S n$ converts to an amorphous phase. When we measured the EXAFS of the $20 \mathrm{vol}$. \% Sn sample as a function of temperature, it was observed that there was essentially no change in EXAFS (Figure 3 ) as the sample was heated above the $\mathrm{Sn}$ melting point, and the thermal damping was much less than for $\beta-\mathrm{Sn}$. This indicates that the $\mathrm{Sn}$ phase is stable at higher temperatures.

We have analysed our experimental data using EXAFS fitting techniques based on the University of Washington software package. Fourier filtered EXAFS of the samples were used for fitting using a model containing $\mathrm{Sn}-\mathrm{Sn}$ and $\mathrm{Sn}-\mathrm{Ge}$ bonds. Measured EXAFS of $\beta$-Sn was used as a standard. The contamination of the $\alpha$-Sn standard with $\beta$-Sn made it difficult to use. Therefore, first shell EXAFS corresponding to $\alpha$-Sn was calculated using the FEFF theoretical code which takes into account the curved wave nature of the photoelectron and multiple-scattering contributions. [4] A similar approach was made to calculate the first shell EXAFS contribution from an ordered $\alpha-\mathrm{Sn} / \mathrm{Ge}$ alloy which was also used as a Sn-Ge standard in the fitting procedure. Initial results indicate that this

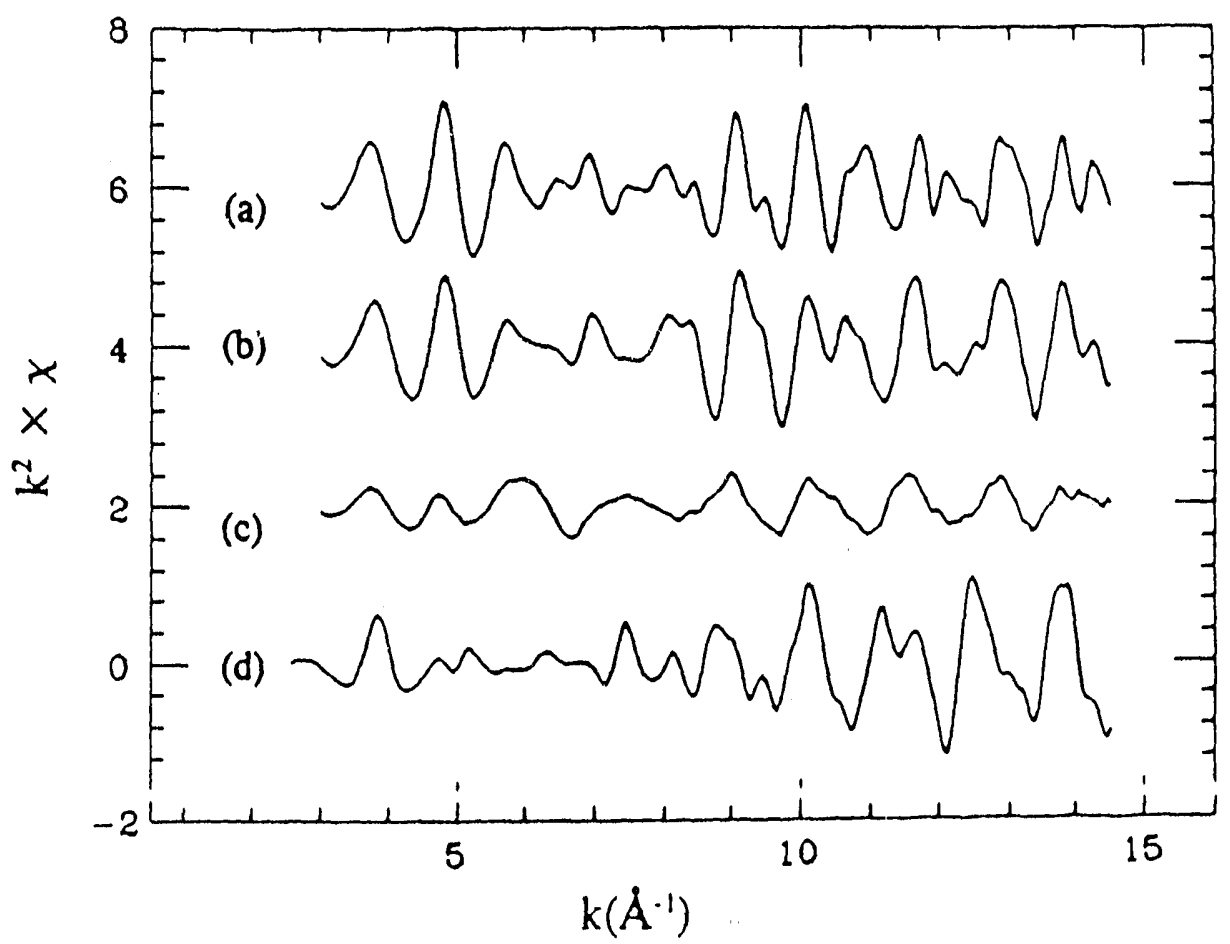

Fig.1 Sn EXAFS data of samples (a) $\beta-S n$ (b) 50 vol.\% Sn (c) 20 vol. $\% \mathrm{Sn}$ and (d) $\alpha$-Sn measured at $10^{\circ} \mathrm{K}$. 


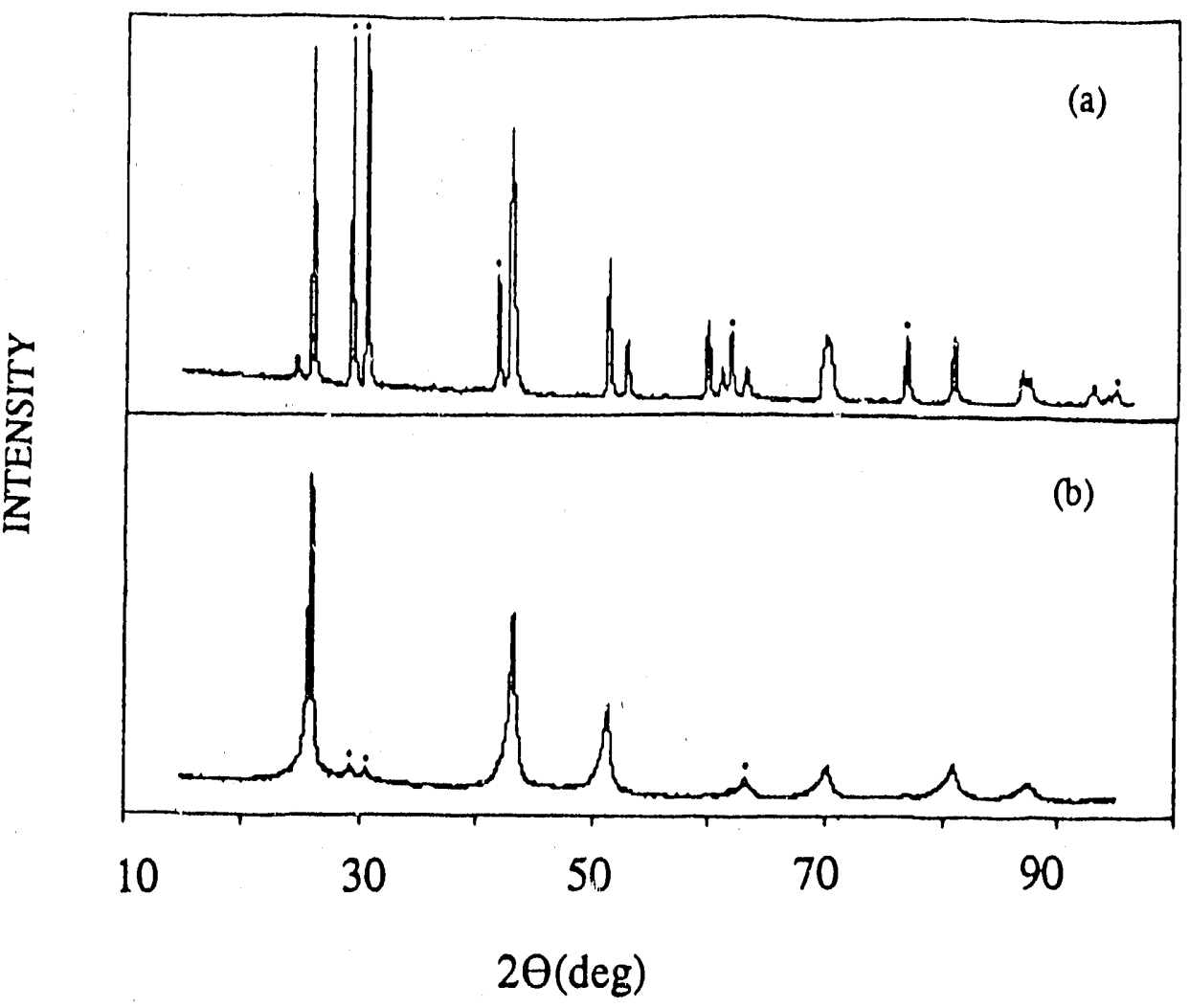

Fig. $2 \mathrm{X}$-ray diffraction data taken (at $8 \mathrm{keV}$ ) at room temperature for samples, (a) $50 \mathrm{vol} . \% \mathrm{Sn}$ and (b) 20 vol.\% Sn. (Peaks with a dot above correspond to $\beta-\mathrm{Sn}$.)

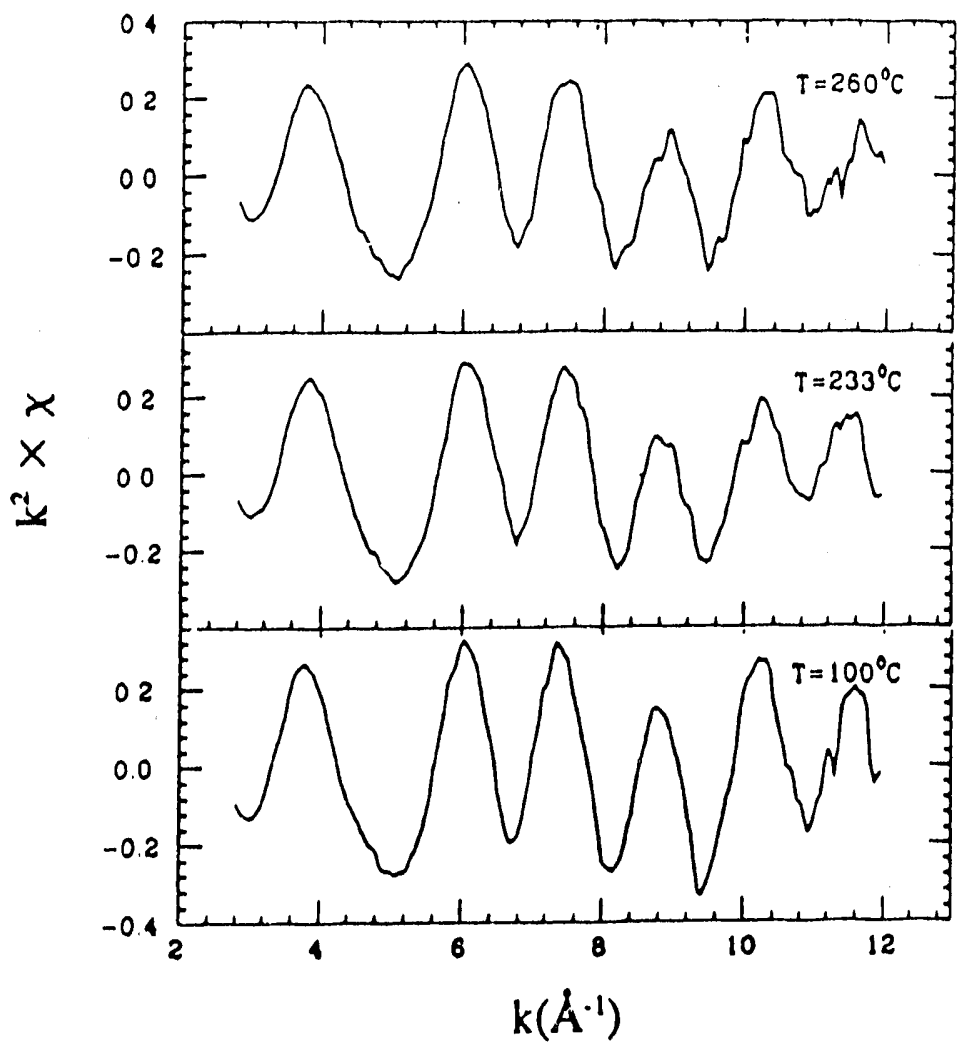

Fig.3 Temperature dependant EXAFS of 20 vol.\% Sn sample. (No significant change in EXAFS amplitude is seen.) 
amorphous form is due to the formation of a disordered phase similar to an $\alpha-S n / G e$ alloy. Fitting gives a $\mathrm{Sn}-\mathrm{Sn}$ bond distance of $2.74 \pm .03 \AA$ and a $\mathrm{Sn}-\mathrm{Ge}$ bond distance of $2.60 \pm .03 \AA$. The $\mathrm{Sn}-\mathrm{Sn}$ bond distance is similar to that of $\alpha$-Sn $(2.81 \AA)$. If we calculate the lattice parameter of a cubic 50:50 $\alpha-\mathrm{Sn} / \mathrm{Ge}$ alloy using the corresponding lattice parameters of $\alpha-\mathrm{Sn}$ and $\mathrm{Ge}(6.46 \AA$ and $5.65 \AA$ respectively[5]) we obtain an average near neighbor distance of $2.62 \AA$ which is in reasonable agreement with our fitting result. We can visualize this as an alloy formed by interfacial Sn going into the Ge structure. The alloy:total $\mathrm{Sn}$ ratio is found to increase from approximately $40 \mathrm{wt} . \%$ tn about $100 \mathrm{wt}$. \% with the decreasing $\mathrm{Sn}$ content. The broadening of Ge $\mathrm{x}$-ray diffraction peaks (Figure 2) with the decreasing $\mathrm{Sn}$ content implies that the decreasing particle size makes more interfacial area available for alloying. XRD line shape analysis shows that the Ge particle size decreases from about $70 \mathrm{~nm}$ to about $15 \mathrm{~nm}$ when going from 50 to 20 vol. \% Sn sample. The observed temperature stability of the $20 \mathrm{vol}$. \% Sn sample above the melting point of bulk Sn may be due to the formation of this alloy. It is reported that the epitaxial films of 50:50 $\alpha-\mathrm{Sn} / \mathrm{Ge}$ alloys can be grown on $\mathrm{Ge}$ substrates at temperatures up to $400^{\circ} \mathrm{C}$.[6] This also explains the decrease in melting enthalpy of $\mathrm{Sn}$ observed by Jang and Koch as the $\mathrm{Sn}$ content is decreased. When essentially all available $\mathrm{Sn}$ (when all $\mathrm{Sn}$ becomes interfacial) participates in alloying, the melting enthalpy would vanish as they have observed.

To summarize, based on both EXAFS and $x$-ray diffraction results, the disorder at the $\mathrm{Sn}$-Ge interface may be attributed to the formation of an $\alpha-\mathrm{Sn} / \mathrm{Ge}$ alloy. The observed stability of this alloy above the melting point of bulk $\mathrm{Sn}$ explains the behavior of melting enthalpy of $\mathrm{Sn}$ in milled $\mathrm{Sn}-\mathrm{Ge}$ powders, as the Sn content is decreased.

\section{ACKNOWLEDGEMENTS}

We would like to thank Dr C. K. Saw of Hoechest-Celanese and Dr. S. S. Prasad of Moltech Corp. for their help with various parts of this experiment. This work is supported by the U. S. Department of Energy, Division of Material Sciences under contract no. DE-AC02-76CH00016. The $X-11$ beamlines are also supported by the DOE Division of Material Sciences under contract no. DEAS05-80-ER10742.

\section{REFERENCES}

1.C.C. Koch, J.S.C. Jang and S.S. Gross, J.Mater.Res., 4, 557 (1989).

2.J.S.C. Jang and C.C. Koch, J.Mater.Res., 5, 325 (1990).

3.R.W. Smith, Journel of the Less-Common Metals, 114, 69 (1986).

4.J. Mustre, Y. Yacoby, E.A. Stern and J.J. Rehr, Phys. Rev. B i2, 10843 (1990).

5.C Kittel, Introduction to Solid State Physics, 6th ed. (John Wiley \& Sons, New York, 1986), p.20.

6.H. Hochst, M.A. Engelhardt and I. Hernandez-Calderon, Phys. Rev B 40, 9703 (1989).

\section{DISCLAIMER}

\footnotetext{
This report was prepared as an account of work sponsored by an agency of the United States Government. Neither the United States Government nor any agency thereof, nor any of their employees, makes any warranty, express or implied, or assumes any legal liability or responsibility for the accuracy, completeness, or usefulness of any information, apparatus, product, or process disclosed, or represents that its use would not infringe privately owned rights. Reference herein to any specific commercial product, process, or service by trade name, trademark, manufacturer, or otherwise does not necessarily constitute or imply its endorsement, recommendation, or favoring by the United States Government or any agency thereof. The views and opinions of authors expressed herein do not necessarily state or reflect those of the United States Government or any agency thereof.
} 

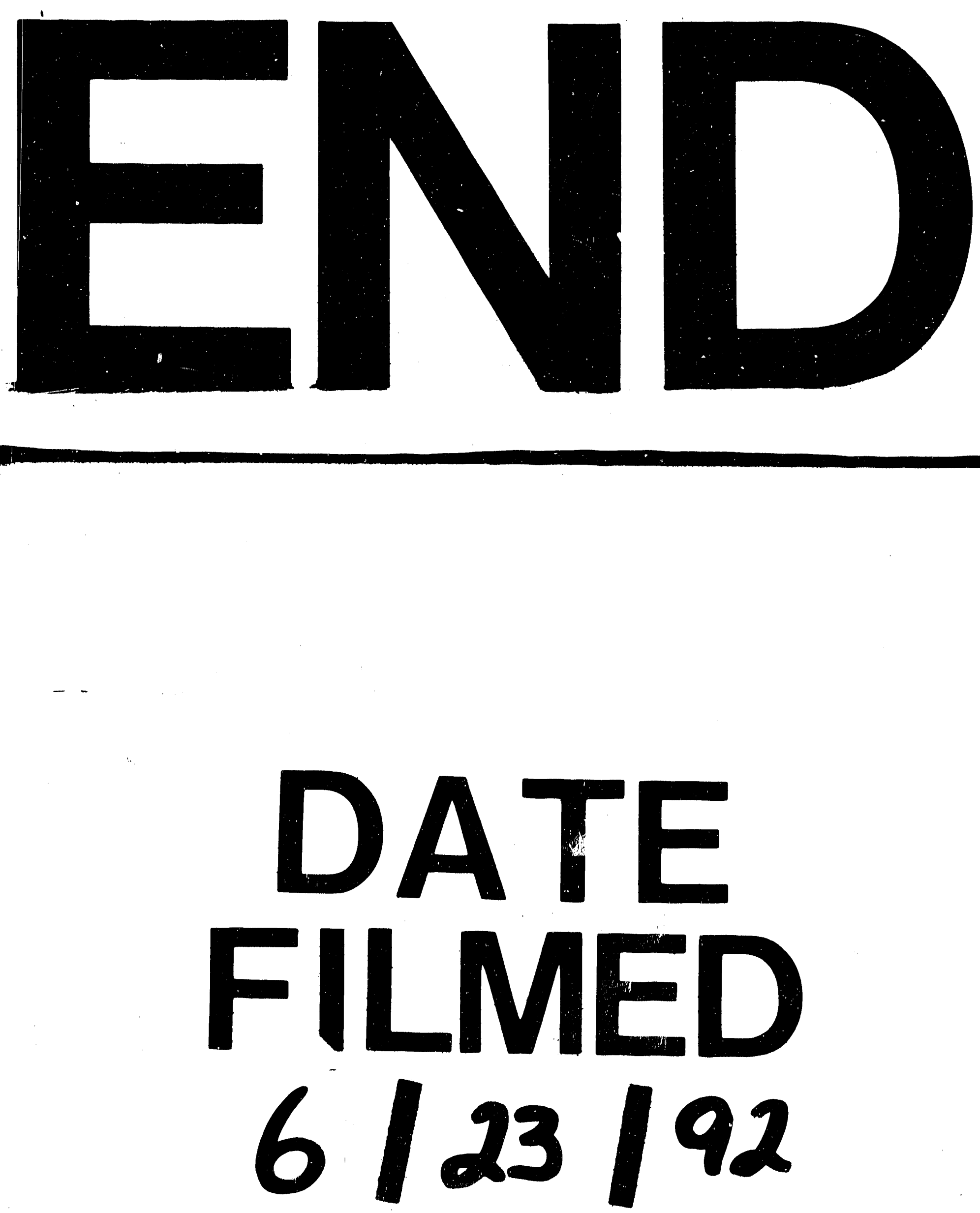
